turbed about every half-hour by a scalding sensation down the penis, accompanied each time by a flow of urine down the tube, each paroxysm lasting about two minutes. - 19th : Still troubled with scalding down the urethra about every hour. and lasting about two minutes. These attarks have not, however, interfered much with his sleep. The drawsheets were stained with distinct areas of clear urine, bloodclot, and mucus. - 20th: The patient is much better to-day, and considers that he is no worse than immediately previous to the operation. After this the patient found great benefit from drinking Bethesda water, aud the washing out of the bladder twice daily with a solution of vitrate of silver (four grains to the ounce) - 26th : The patient is enabled to walk out and enjoy himself, and only suffers from such hæmorrhage as may be reasonably attributed to the raw sur$f$, ce of a perineal sinus which still remains. -July $16 \mathrm{tb}, 1883$ The patient is convalescent and free from hemorrhage, and a sle to go about much as usual.

(To be concluded.)

\section{EMETICS FOR BONES IN THE THROAT.}

\section{BX JAMES GREY GLOVER, M.D. \&c.}

IT is curious that in several of the best text-books on surgery, among the means used for dislodging bodies in the throat, no mention is made of emetics. A few months ago $I$ had to make the discovery of their value in the following circumstances.

On a Sunday morning, at about 10 o'clock, a gentleman asked me to see his wife, who the previous day, about 2 o'clock, in taking some soup, became conscious that she had swallowed a piece of mutton bone, which quickly went so far down that it could not be felt by a medical friend whom she saw shortly afterwards. When I saw her, about twenty hours after the occurrence, I was equally unable to feel the foreign body, but the distress of the lady left no doubt of the presence of something in the passage exceedingly disagreeable. Swallowing was only accomplished with the most painful effort and in the smallest quantities. I had, fortunately, no forceps with me, and the case was urgent. It occurred to me that if $I$ could reverse the action of the stomach and cosophagus by an emetic, I might procure the object in view, and save the necessity for using instruments blindly. I al ways carry a drachm of sulphate of zinc in my purse. The diffically was to get the patient to swallow with any satisfactory quantity of fluid. It was only by dint of great and painful effort on her part, at my urgent request, that she managed to take half a drachm in a fair amount of water. As she was not predisposed to sickness, and at the end of five minutes had no sensation of the sort, I gave her a second half drachm with more warm water. In a few minutes free vomiting began, and the first act of vomiting was followed by a chink in the vessel and the presence in it of a piece of mutton bone of the form and size represented below, and with four sharp edges and four (especially two) sharp points.

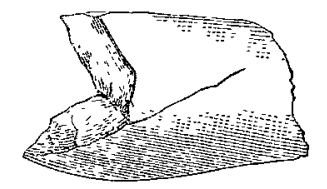

It can scarcely be doubted that a body of quadrilateral form, with such angles and edges, could only have been pushed downwards mechanically with serious injury to the walls of the osophagus. Supposing it to have been placed horizontally or nearly so, as, from the difficulty of passage, would seem to have been the case, its seizure and removal by forceps would have been scarcely less hazardous. Nothing, on the other hand, could have been more happy than the effect of the emetic. A trifling soreness for a day or two was the only unpleasant consequence of the twenty hours' impaction.

Shortly after this case I had two others of fishbone in the throat. These were both small, and in neither could the bone be felt with a short index finger. In both I had recourse to an emetic with prompt and effectual relief.

I have since found that emetics have been recommended by various authorities in these circumstances. An instance of the use of one and a quarter milligramme of apomorpbia hypodermically, followed closely by a second dose, in a child aged ten, and in whom not even water could $p \neq$ ss the obstruct.on caused by a plum-stone in the ceopbagus, is quoted at page 504 of the Medical Times and Gazette for Out. 26th, 1575. In less than two minutes free vomiting and the expulsion of the stone followed. Irresistible sleep also fol. lowed, which was overcome by strong coffee. A pomorphia may be safely used hypodermically in case of adults where other emetics cannot be swallowed; but the sulphate of zinc with water will generally ans wer where emetics are indicated. The more water the better and safer is their action likely to be. Highbury.

\section{ON THE TREATMENT OF MEASIES.}

BY D. MACLEAN, M.D. GLAS.,

PHYSICILN TO THE GLASGOW PULLIC DISPENSARY, CHEST AND THROAT CLINIQUE

$I_{N}$ the present prevalence of this disease it may not be out of place to call attention to a method of treatment which has been found, in my hands, of what might be called universal success. Considering that this disease is one that must be passed through by almost all members of the human family at some period of their existence, we may take it for granted that it is something essential that the human frame should undergo for the purpose of perfect health. Taking also for granted that it is the canse of cutting short many lives, it behoves us to take advantage of every means that can abbreviate the duration of the disease and diminish the mortality which so unhappily springs fromit. We see every now and again in the journals and newspapers that an epidemic of measles has taken place in such and such a quarter, and that it was necessary to close the schools, \&c. Nor is it only in our own country that we learn of so many deaths taking place, but we read of how its fearful ravages decimate whole populations, such as have recently taken place in Fiji. It thus seems essential that any form of treatment (for we cannot apparently prevent the onset) that can diminish the mortality must be of paramount importance whenever these epidemic or even sporadic cases appear. The form of treatment which I propose to lay betore the members of our profession, and the remedies which I have been in the habit of using for a good many years, will enable them to try the same in their own practices, and ultimately refute or corroborate the conclusions I have long ago arrived at.

As this disease is considered one of the zymotic class, we have in its treatment, to consider, principally two things(1) the management of the ferment, or whatever it is; and (2) the management of the effects of this ferment upon the system. The most marked of these latter present themselves to us in the effects of the ailment upon the mucous membranes. The greatest action of the disease, as we all know, is upon the mucous membrane of the lungs, and it is trom its action there we have the immediate cause of the ensuing death, or the prolonged ill-health afterwards. We have thus clearly set before us the line of action to follow :-(1) To relieve the congestion of the mucous membrane, which is the immediate cause of danger; and (2) to destroy or reduce the violence of the disease itself. This I have been in the habit of doing, I believe successfully, by giving (say to a child of two or three years of age) a teaspoonful in water of the following mixture every three hisurs:-Ipeca. cuanha wine, half a drachm; syrup of squills, half an ounce; quinine, two grains; acetate of ammonia solution to two ounces. Of course the quinine is increased according to age We have thus in this mixture a simulating expectorant and diaphoretic to relieve the tension in the mucuns membranes and the skin, and also in the quinine a specific to destroy or abate the violence of the primary ferment. It may be neces sary to add to or modify the form in which this plan of treat. ment is carried out; as when the irritation and cough are persistently great, then the addition of a little tiucture of hyoscyamus is all that is necessary. So with the quinine; sometimes the stomach is so irritable that it is necessary to omit it from the mixtare; but as it is essential that it be introduced into the system for the destruction of the ferment, it can be administered separately by giving it in powder, mixed with saccharated carbonate of iron, which 